\title{
Characterization of Markovian equilibria in a class of differential games
}

\author{
J.P. Rincón-Zapatero* \\ Department of Applied Economics (Mathematics), University of Valladolid, Avda. Valle Esgueva 6, \\ 47011 Valladolid, Spain
}

\begin{abstract}
We consider an alternative method to the classical one for the determination of Markov perfect Nash equilibria. The approach shown in the paper is based on the study of a quasi-linear system of partial differential equations instead of the Hamilton-Jacobi-Bellman system. The simpler structure of the former allows us to determine existence and uniqueness of Nash equilibria in non-renewable resource games under some assumptions. When closed-form solutions are not available, we give a method to obtain numerical solutions.
\end{abstract}

JEL classification: $\mathrm{C} 73 ; \mathrm{Q} 32$

Keywords: Differential games; Markovian equilibrium; Quasi-linear equation; Non-renewable resource

\section{Introduction}

The development of game theory has been spectacular in the recent years. In particular, one of the more promising discipline is differential game theory. In this framework, it is supposed that interactions among rational agents take place instantaneously along time. This facilitates the modelling of problems in biology, economics and engineering, and allows us to obtain robust solutions.

As it is well established in the literature, the most relevant concept of solution in a non-coperative game is Nash equilibrium. Generally speaking, open-loop strategies, which are programmed for each player from the beginning of the game, lead to suboptimal decisions. Therefore, the Nash equilibrium obtained with these type of strategies is not subgame perfect. On the other hand, with (non-stationary) Markov rules the

\footnotetext{
* Corresponding author. Tel.: +34-983 186 591; fax: +34-983 423299.

E-mail address: zapatero@eco.uva.es (J.P. Rincón-Zapatero).
} 
decision of each player depends on time and the present value of the state variable. This fact makes it possible for Nash equilibrium be subgame perfect, in such a way that the players do not have incentives to deviate unilaterally at any stage of the game.

Traditionally, the characterization of Markovian equilibrium depends on the HamiltonJacobi-Bellman (HJB) system of partial differential equations; see Friedman (1971) and Başar and Olsder (1999). It is well known in the literature that if the value function of each player is smooth enough, then it is a solution to the HJB system. However, the system is in general non-linear. This fact can make its analytical as well as numerical studies difficult.

Our aim in this paper is to provide an alternative method for the study of a class of differential games that are of relevance in some applications in Economics, as for example, games of resource extraction. This new approach - which is, however, closely related with the HJB system - was proposed in Rincón-Zapatero et al. (1998) for the smooth case. There, a quasi-linear system of partial differential equations characterizing Markov perfect Nash equilibrium (MPNE) was found using the Pontryagin's maximum principle. The scope of the approach was limited to games where the dimension of the state space equals the number of controls of each player, and the MPNE is interior to the control region. Nevertheless, we were able to establish the existence of MPNE in rather general differential games of non-renewable resources in finite horizon.

The main objective of this paper is to extend the results in Rincón-Zapatero et al. (1998) to a non-smooth setting. We consider continuous and piecewise smooth Markovian strategies and give sufficient conditions for their optimality. These sufficient conditions are critically based on the following assumptions: (i) each player's control space has the same dimension as the state space of the system, and the control region is unconstrained; (ii) the region of non-smooth points of each player's control is contained in a finite union of curves in the graph of trajectories; (iii) the state equations have a unique solution for each initial condition when piecewise-continuous feedback controls are used; and (iv) the trajectory meets the region of non-smoothness at most a finite number of points.

The special structure of the quasi-linear equations gives more information than that of a general equation of first order. This fact is exploited in this paper to obtain new results on the joint exploitation of a non-renewable resource in a competitive framework.

In Section 2, we present the types of differential games we consider and the definition of MPNE. We also review some of the results from Rincón-Zapatero et al. (1998). In Section 3, we establish the main result of the paper. We give sufficient conditions which imply that a continuous and piecewise smooth solution of a quasi-linear system of PDEs is an MPNE. In Section 4, we apply the results obtained in the previous section to the study of symmetric, non-renewable resource games. In particular, we prove the existence and uniqueness of MPNE when the discount rate is zero. We are also able to find closed-form solutions in this case and perform a sensitivity analysis of the solution with respect to variations in the number of players, residual function and the intertemporal rate of substitution. The classical result that Markovian equilibria are inefficient is corroborated. We also include a numerical method - well known in 
the literature of quasi-linear equations - demonstrating the usefulness of our approach. We conclude with the additional remarks of Section 5 and an Appendix.

\section{Game description and preliminary results}

We consider an $N$-person differential game over a fixed time interval,

$$
\begin{aligned}
& \max _{u^{i}}\left\{J^{i}\left(x_{0}, t_{0}, u^{1}, \ldots, u^{N}\right)=\int_{t_{0}}^{T} L^{i}\left(x, t, u^{1}, \ldots, u^{N}\right) \mathrm{d} t+S^{i}(x(T), T)\right\}, \\
& \text { s.t. } \dot{x}=f\left(x, t, u^{1}, \ldots, u^{N}\right), \quad x\left(t_{0}\right)=x_{0}, \\
& u^{i}(t) \in U^{i}, \quad \forall t \in\left[t_{0}, T\right], \quad U^{i} \text { a subset of } \mathbb{R}^{n}
\end{aligned}
$$

for $i=1, \ldots, N$. Here the functions $L^{i}, f$ and $S^{i}$ are assumed to be twice continuously differentiable. The vectors $x=\left(x_{1}, \ldots, x_{n}\right)^{\mathrm{T}} \in \mathbb{R}^{n}$ and $u^{i}=\left(u_{1}^{i}, \ldots, u_{n}^{i}\right)^{\mathrm{T}} \in \mathbb{R}^{n}$ represent the state and control variables for the $i$ th player, respectively, where the superscript $\mathrm{T}$ denotes the 'transpose'. The function $f$ satisfies a uniform Lipschitz condition with respect to $\left(u^{1}, \ldots, u^{N}\right)$ and has linear growth with respect to $x$. We associate with each $u^{i}$ a continuous and piecewise smooth function $\phi^{i}: \mathbb{R}^{n}\left[t_{0}, T\right] \rightarrow U^{i}$ such that $u^{i}(t)=$ $\phi^{i}(x(t), t)$ and the dynamics (1) admits a unique solution. Hence we are considering Markov strategies for the players. We denote by $\mathscr{U}^{i}$ the set of functions $\phi^{i}$ satisfying the above conditions and let $\mathscr{U}=\mathscr{U}^{1} \times \cdots \mathscr{U}^{N}$. We refer to $\mathscr{U}^{i}$ as the set of admissible controls of player $i$ and to $\mathscr{U}$ as the set of admissible profiles.

The system

$$
\dot{x}=f\left(x, t, \phi^{1}(x, t), \ldots, \phi^{N}(x, t)\right), \quad x\left(t_{0}\right)=x_{0}
$$

is the closed-loop system associated to the profile $\phi=\left(\phi^{1}, \ldots, \phi^{N}\right)$. Let us denote by $y^{\phi}(s ; x, t), t \leqslant s \leqslant T$ the (unique) trajectory associated with the profile $\phi$ when the initial condition is $(x, t)$. Obviously $y^{\phi}(t ; x, t)=x$.

An $N$-tuple of strategies $\hat{\phi} \in \mathscr{U}$ is an MPNE of the game if, for all $i=1, \ldots, N$,

$$
J^{i}\left(x, t,\left(\phi^{i} \mid \hat{\phi}_{-i}\right)\right) \leqslant J^{i}(x, t, \hat{\phi}), \quad \forall \hat{\phi}^{i} \in \mathscr{U}^{i}, \quad t \in\left[t_{0}, T\right], \quad x \in \mathbb{R}^{n},
$$

where $\hat{\phi}_{-i}=\left(\hat{\phi}^{1}, \ldots, \hat{\phi}^{i-1}, \hat{\phi}^{i+1}, \ldots, \hat{\phi}^{N}\right)$ and $\left(\phi^{i} \mid \hat{\phi}_{-i}\right)=\left(\hat{\phi}^{1}, \ldots, \hat{\phi}^{i-1}, \hat{\phi}^{i}, \hat{\phi}^{i+1}, \ldots, \hat{\phi}^{N}\right)$.

By definition, an MPNE is a Nash equilibrium whatever the initial condition is. Hence, if the players play the MPNE profile, then no player should have any incentive, at any future stage of the game, to deviate unilaterally from this recommendation. In contradistinction with the Nash equilibrium based on open-loop controls, the equilibrium found with Markov controls allows us to instantaneously update the decisions of the agents by means of the value of the state variable. In this way, Nash equilibrium may enjoy the property of subgame perfection. However, it is important to note that not all Nash equilibria based on Markov strategies are necessarily subgame perfect.

The standard approach adopted in the literature to determine MPNE is to solve the HJB system of PDEs; see Friedman (1971) and Başar and Olsder (1999). In general, 
this system is non-linear, so its study and resolution are not easy, both from analytical and computational points of view. The HJB system is

$$
\begin{aligned}
& V_{t}^{i}+H^{i}\left(x, t, u_{0}^{1}\left(x, t, \nabla_{x} V^{1}\right), \ldots, u_{0}^{N}\left(x, t, \nabla_{x} V^{N}\right)\right)=0, \quad t_{0} \leqslant t<T, \\
& V^{i}(x, T)=S^{i}(x), \quad x \in \mathbb{R}^{n},
\end{aligned}
$$

$i=1, \ldots, N$, where $H^{i}$ is the Hamiltonian of the $i$ th player,

$$
H^{i}\left(x, t, u^{1}, \ldots, u^{N}, \lambda^{i}\right)=L^{i}\left(x, t, u^{1}, \ldots, u^{N}\right)+f^{\mathrm{T}}\left(x, t, u^{1}, \ldots, u^{N}\right) \lambda^{i}
$$

with $\lambda^{i}$ being his or her costate variable. The functions $u^{i}=u_{0}^{i}\left(x, t, \lambda^{i}\right)$ are assumed to be of class $\mathscr{C}^{1}$, and obtained from

$$
H^{i}\left(x, t, u_{0}^{1}\left(x, t, \lambda^{1}\right), \ldots, u_{0}^{N}\left(x, t, \lambda^{N}\right), \lambda^{i}\right)=\max _{u^{i} \in U^{i}} H^{i}\left(x, t,\left(u^{i} \mid\left(u_{0}\right)_{-i}\right), \lambda_{i}\right) .
$$

A classical result of Friedman (1971, Theorem 8.2.3) states that if there is a solution $V$ to (2)-(3), of class $\mathscr{C}^{2}$, then $\phi(x, t)=u_{0}^{i}\left(x, t,\left(\nabla_{x} V^{1}, \ldots, \nabla_{x} V^{N}\right)\right)$ is of class $\mathscr{C}^{1}$ and $\phi=\left(\phi^{1}, \ldots, \phi^{N}\right)$ is an MPNE.

In Rincón-Zapatero et al. (1998), we discussed another method to characterize MPNE, based on a system of quasi-linear partial differential equations, intimately related with the HJB system. In the quasi-linear system presented below the unknown is the profile of strategies of the players, whereas in the HJB system the unknown is the vector of value functions of the players. However, the HJB approach applies without restrictions on the dimensions of the controls and state variables, and allow us to handle also constraints on the control variables.

In order to make the exposition more transparent, in the following we take $n=1$, although the results also hold without this assumption whenever the number of controls of each player coincide with the dimension of the state space. By means of the application of Pontryagin's maximum principle, in Rincón-Zapatero et al. (1998) it is proved that a smooth and interior MPNE must satisfy the following system of partial differential equations:

$$
\begin{aligned}
& \hat{H}_{u^{i} t}^{i}+\hat{H}_{u^{i} x}^{i} f+\sum_{j=1}^{N} \hat{H}_{u^{i} w^{j}}^{i}\left(\hat{\phi}_{t}^{j}+\hat{\phi}_{x}^{j} f\right)+\hat{H}_{u^{i} \lambda^{i}}^{i}\left(-\hat{H}_{x}^{i}-\sum_{j=1}^{N} \hat{H}_{u^{j}}^{i} \hat{\phi}_{x}^{j}\right)=0, \\
& \quad i=1, \ldots, N,
\end{aligned}
$$

where $\hat{H}_{\{\cdot\}}^{i}(x, t, u)$ denotes $H_{\{\cdot\}}^{i}$ evaluated at $\left(x, t, \hat{\phi},-\left(L_{u^{i}}^{i} / f_{u^{i}}\right)(x, t, \hat{\phi})\right)$, and a variable in subscript notation stands for partial differentiation. In Eq. (4), the independent variables are $x, t$ and the dependent ones are $\left(\hat{\phi}^{1}, \ldots, \hat{\phi}^{N}\right)$. In the system above the costate variable of each player, $\lambda^{i}$, has been substituted by $-L_{u^{i}}^{i} / f_{u^{i}}$. Of course, we are assuming that $f_{u^{i}} \neq 0$.

The transversality condition established by the maximum principle and the maximization of the Hamiltonian function provides a set of final conditions for system (4) given by

$$
L_{u^{i}}^{i}(x, T, \hat{\phi})+f_{u^{i}}(x, T, \hat{\phi}) S_{x}^{i}(x, T)=0, \quad i=1, \ldots, N .
$$


Definition 1. Suppose that for all $i=1, \ldots, N, f_{u^{i}} \neq 0$. We say that $\hat{\phi} \in \mathscr{U}$ satisfies the Nash property if

$$
H^{i}\left(x, t,\left(\phi^{i} \mid \hat{\phi}_{-i}\right), \Gamma^{i}(x, t, \hat{\phi})\right) \leqslant H^{i}\left(x, t, \hat{\phi}, \Gamma^{i}(x, t, \hat{\phi})\right)
$$

for all $t \in\left[t_{0}, T\right], x \in \mathbb{R}, i=1, \ldots, N$ and for all $\phi^{i} \in \mathscr{U}^{i}$, where

$$
\Gamma^{i}(x, t, u)=-f_{u^{i}}^{-1}(x, t, u) L_{u^{i}}^{i}(x, t, u) .
$$

Remark 1. If $U^{i}$ is convex and the Hamiltonians of the players are concave with respect to their own control variable $u^{i}$, then any profile that is interior to $U$ has the Nash property.

In Rincón-Zapatero et al. (1998), it was proved that a $\mathscr{C}^{1}$ solution of (4), (5) being an admissible profile of strategies and verifying the Nash property, is an MPNE.

\section{Main result}

In this section, we will extend the sufficient condition established in the previous section to non-smooth solutions of (4), (5). The value function of each player is defined as

$$
\begin{aligned}
& V^{i}(x, t)=\max \left\{\int_{t}^{T} L^{i}\left(y, s,\left(\phi^{i} \hat{\phi}_{-i}\right)\right) \mathrm{d} s+S^{i}(y(T), T):\right. \\
&\left.\dot{y}=f\left(y, s,\left(\phi^{i} \mid \hat{\phi}_{-i}\right)\right), \quad s \in(t, T) ; \quad y(t)=x, \quad \phi^{i} \in \mathscr{U}^{i}\right\} .
\end{aligned}
$$

Theorem 1. Let $\hat{\phi} \in \mathscr{U}$ be a continuous and piecewise smooth solution to (4), (5) having the Nash property, such that $f_{u^{i}}(x, t, \hat{\phi}) \neq 0$. Suppose further that the region of non-smoothness of $\hat{\phi}^{i}$ is a finite union of curves: $C_{j}^{i}=\left\{(x, t): \theta_{j}^{i}(x, t)=0\right\}, i=1, \ldots, N$, $j=1, \ldots, m_{i}$, where

(i) $\theta_{j}^{i}: \mathbb{R} \times\left[t_{0}, T\right] \rightarrow \mathbb{R}$ is $\mathscr{C}^{1}$ and $\theta_{j x}^{i}(x, t) f(x, t, \hat{\phi})+\theta_{j t}^{i}(x, t) \neq 0$ for all $(x, t) \in \mathbb{R} \times$ $\left[t_{0}, T\right]$.

(ii) There exists a unique solution $y^{\hat{\phi}}(s ; x, t)$ of the dynamics for all initial conditions $(x, t)$; this trajectory meets the set $C=\bigcup_{i=1}^{N} \bigcup_{j=1}^{m_{i}} C_{j}^{i}$ at most at a finite number of points.

Then, $\hat{\phi}$ is an MPNE. Furthermore, the value function of each player satisfies

$$
V_{x}^{i}(x, t)=\Gamma^{i}(x, t, \hat{\phi}(x, t)), \quad i=1, \ldots, N .
$$

Proof. The idea of the proof is to show that the costate variable of each player equals the partial derivative of his value function with respect to the initial value of the state variable. Given an initial condition $(x, t) \notin C$, let us denote by $\tau_{j}^{i}(x, t)$ a time point when $y^{\hat{\phi}}(s ; x, t)$ meets $C_{j}^{i}$. An induction argument with respect to $i, j$ easily shows that $y^{\hat{\phi}}$ is $\mathscr{C}^{1}$ with respect to $(s, x, t)$. To proceed further, we note that on the one hand 
$\theta_{j}^{i}\left(\tau_{j}^{i}(x, t), y^{\hat{\phi}}\left(\tau_{j}^{i}(x, t) ; x, t\right)\right)=0$ by definition and, on the other hand, (i) implies

$$
\frac{\mathrm{d}}{\mathrm{d} s} \theta_{j}^{i}\left(y^{\hat{\phi}}(s ; x, t), s\right)=\theta_{j x}^{i}\left(\mathrm{~d} y^{\hat{\phi}} / \mathrm{d} s\right)+\theta_{j s}^{i}=\theta_{j x}^{i} f+\theta_{j s}^{i} \neq 0 .
$$

Applying the Implicit Function Theorem to the equation

$$
\theta_{j}^{i}\left(y^{\hat{\phi}}(s ; x, t), s\right)=0,
$$

we arrive at the conclusion that (6) admits a $\mathscr{C}^{1}$ local solution $s=\tau_{j}^{i}(x, t)$. Thus the composition $y^{\hat{\phi}}\left(\tau_{j}^{i}(x, t) ; x, t\right)$ is also smooth. Hence we can differentiate it with respect to $x$.

Now let us denote the instants of time where $y^{\hat{\phi}}$ intercepts $C$ by $t=\tau_{0}<\tau_{1}<\cdots<$ $\tau_{q}<\tau_{q+1}=T$. We have

$$
\begin{aligned}
J^{i}(x, t, \hat{\phi}) & =\int_{t}^{T} L^{i}\left(y^{\hat{\phi}}(s ; x, t), s, \hat{\phi}\left(y^{\hat{\phi}}(s ; x, t), s\right)\right) \mathrm{d} s+S^{i}\left(y^{\hat{\phi}}(T ; x, t), T\right) \\
& =\sum_{n=0}^{q}\left(\int_{\tau_{n}}^{\tau_{n+1}} L^{i}\left(y^{\hat{\phi}}(s ; x, t), s, \hat{\phi}\left(y^{\hat{\phi}}(s ; x, t), s\right)\right) \mathrm{d} s\right)+S^{i}\left(y^{\hat{\phi}}(T ; x, t), T\right) .
\end{aligned}
$$

We can differentiate under integral sign to obtain

$$
\begin{aligned}
J_{x}^{i}(x, t, \hat{\phi})= & \sum_{n=0}^{q}\left(\int_{\tau_{n}}^{\tau_{n+1}} \frac{\partial}{\partial x} L^{i}\left(y^{\hat{\phi}}(s ; x, t), s, \hat{\phi}\left(y^{\hat{\phi}}(s ; x, t), s\right)\right) \mathrm{d} s\right. \\
& +L^{i}\left(\tau_{n+1}, y^{\hat{\phi}}\left(\tau_{n+1} ; x, t\right), \hat{\phi}\left(\tau_{n+1}, y^{\hat{\phi}}\left(\tau_{n+1} ; x, t\right)\right)\right) \frac{\partial}{\partial x} \tau_{n+1} \\
& \left.-L^{i}\left(y^{\hat{\phi}}\left(\tau_{n} ; x, t\right), \tau_{n}, \hat{\phi}\left(y^{\hat{\phi}}\left(\tau_{n} ; x, t\right), \tau_{n}\right)\right) \frac{\partial}{\partial x} \tau_{n}\right) \\
& +\frac{\partial}{\partial x} S^{i}\left(y^{\hat{\phi}}(T ; x, t), T\right) .
\end{aligned}
$$

As $(\partial / \partial x) \tau_{0}=(\partial / \partial x) \tau_{q+1}=0$ and $\hat{\phi}$ is continuous, we have

$$
J_{x}^{i}(x, t, \hat{\phi})=\int_{t}^{T} \frac{\partial}{\partial x} L^{i}\left(y^{\hat{\phi}}(s ; x, t), s, \hat{\phi}\left(y^{\hat{\phi}}(s ; x, t)\right), s\right) \mathrm{d} s+\frac{\partial}{\partial x} S^{i}\left(y^{\hat{\phi}}(T ; x, t), T\right) .
$$

Now, as it is shown in the proof of Theorem 4.1 in Rincón-Zapatero et al. (1998), the last term in the above equality coincides with $\Gamma^{i}(x, t, \hat{\phi})$. It is at this point that we use the fact that $\hat{\phi}$ is a solution of (4), (5) when it is smooth.

To continue with the proof, let us denote by $y(s)$ the trajectory associated with the profile $\phi=\left(\phi^{i} \mid \hat{\phi}_{-i}\right)$, where $\phi^{i} \in \mathscr{U}^{i}$ is arbitrary, and with initial condition $(x, t)$. It then follows that

$$
\frac{\mathrm{d}}{\mathrm{d} s} J^{i}(y(s), s, \phi)=J_{t}^{i}(y(s), s, \phi)+J_{x}^{i}(y(s), s, \phi) f(y(s), s, \phi)
$$


and, by the definition of $J^{i}, J_{t}^{i}(y(s), s, \phi)=-L^{i}(y(s), s, \phi)$, so we have the identity

$$
J_{t}^{i}(y(s), s, \phi)+J_{x}^{i}(y(s), s, \phi) f(y(s), s, \phi)+L^{i}(y(s), s, \phi)=0 .
$$

Taking in this equation $\phi^{i}=\hat{\phi}^{i}$ and replacing $J_{x}^{i}(y(s), s, \hat{\phi})$ by $\Gamma^{i}(y(s), s, \hat{\phi})$ we have

$$
\begin{aligned}
0 & =J_{t}^{i}(y(s), s, \hat{\phi})+\Gamma^{i}(y(s), s, \hat{\phi}) f(y(s), s, \hat{\phi})+L^{i}(y(s), s, \hat{\phi}) \\
& =J_{t}^{i}(y(s), s, \hat{\phi})+H^{i}(y(s), s, \Gamma(y(s), s, \hat{\phi}), \hat{\phi}) \\
& \geqslant J_{t}^{i}(y(s), s, \hat{\phi})+H^{i}(y(s), s, \Gamma(y(s), s, \hat{\phi}), \phi) \\
& =J_{t}^{i}(y(s), s, \hat{\phi})+\Gamma^{i}(y(s), s, \hat{\phi}) f(y(s), s, \phi)+L^{i}(y(s), s, \phi) \\
& =\frac{\mathrm{d}}{\mathrm{d} s} J^{i}(y(s), s, \hat{\phi})+L^{i}(y(s), s, \phi)
\end{aligned}
$$

with the inequality being due to the Nash property of $\hat{\phi}$. After integration between $t$ and $T$ we obtain the desired inequality $J^{i}(x, t, \hat{\phi}) \geqslant J^{i}(x, t, \phi)$, because $J^{i}(y(T), T, \hat{\phi})=$ $S^{i}(y(T), T)$.

To complete the proof, if $(x, t) \in C$, then it is possible to choose a sequence $\left(x_{n}, t_{n}\right)$ of points not in $C$ converging to $(x, t)$. By continuity, we can take limits in $J_{x}^{i}\left(x_{n}, t_{n}, \hat{\phi}\right)$ to arrive at the desired result.

\section{Remark 2.}

1. There exist results in the literature guaranteeing condition (ii) of the theorem; see Fleming and Rishel (1975). These are in the context of single player games (i.e., optimal control problems) where the controls present surfaces of discontinuity. We provide here a partial extension of these results to $N$-person games.

2. We establish here the connection between the HJB system and the quasi-linear system. We take $n=1$ to simplify the arguments, but the same holds in the multidimensional case as well. Let $\hat{\phi}$ satisfy the hypotheses of Theorem 1 . Then, the value function of each player, $V^{i}$, is given by

$$
V^{i}(x, t)=\int^{x} \Gamma^{i}(z, t, \hat{\phi}(z, t)) \mathrm{d} z, \quad i=1, \ldots, N,
$$

where $\Gamma^{i}$ is defined in Definition 1. It then follows that $V^{i} \in \mathscr{C}^{1,2}$ and $\left(V^{1}, \ldots, V^{N}\right)$ satisfy the HJB equations:

$$
\begin{aligned}
& V_{t}^{i}(x, t)+\max _{u^{i} \in U^{i}}\left\{L^{i}\left(x, t,\left(u^{i} \mid \hat{\phi}_{-i}\right)\right)+V_{x}^{i}(x, t) f\left(x, t,\left(u^{i} \mid \hat{\phi}_{-i}\right)\right)\right\}=0, \\
& V^{i}(x, T)=S^{i}(x, T),
\end{aligned}
$$

which is shown next. It is readily seen that system (4) can be rewritten in the conservative form

$$
\frac{\partial}{\partial t} \Gamma^{i}(x, t, \hat{\phi}(x, t))+\frac{\partial}{\partial x} \mathscr{H}^{i}(x, t, \hat{\phi}(x, t))=0, \quad i=1, \ldots, N,
$$


where $\mathscr{H}^{i}=L^{i}+\Gamma^{i} f$. Integrating with respect to $x$ in (9) and interchanging differentiation and integration, we have

$$
\frac{\partial}{\partial t} \int^{x} \Gamma^{i} \mathrm{~d} z+\mathscr{H}^{i}=V_{t}^{i}+\mathscr{H}^{i}=0,
$$

where we have suppressed the arguments. Hence

$$
V_{t}^{i}(x, t)+L^{i}(x, t, \hat{\phi})+V_{x}^{i}(x, t) f(x, t, \hat{\phi})=0
$$

because $\Gamma^{i}(x, t, \hat{\phi}(x, t))=V_{x}^{i}(x, t)$. But from the Nash property of $\hat{\phi}$,

$$
L^{i}(x, t, \hat{\phi})+V_{x}^{i}(x, t) f(x, t, \hat{\phi}) \geqslant H^{i}\left(x, t,\left(u^{i} \mid \hat{\phi}_{-i}\right), V_{x}^{i}\right)
$$

for all $i=1, \ldots, N$, for all $u^{i} \in U^{i}$. Therefore, (11) and (12) imply (7). The final condition (8) is trivially fulfilled by construction.

Equality (10) admits a useful interpretation. It expresses a balance equation with the following meaning: the time variation of $\int_{a}^{b} \Gamma^{i} \mathrm{~d} z$ is equal to $-\left.\mathscr{H}^{i}\right|_{a} ^{b}$, where $a, b \in \mathbb{R}$. As shown in Theorem $1, \Gamma^{i}$ is the shadow price of the state variable $x$ for player $i$. On the other hand, $\mathscr{H}^{i}$, the maximized Hamiltonian, is the sum of the instantaneous payoff and the future value of $x$ at the 'price' $\Gamma^{i}$, that is to say, the overall payoff due to the MPNE $\hat{\phi}$ by considering present and future effects of playing $\hat{\phi}$. Hence the time variation of the accumulated shadow price in the interval $a \leqslant x \leqslant b$ equals minus the difference between overall payoffs obtained for player $i$ in $[a, b]$.

3. The proof of the theorem shows that $\hat{\phi}$ is also an MPNE with respect to an enlarged class of feedback rules, possibly discontinuous. Let us observe that it is only the continuity of $\hat{\phi}$ that is used in the proof. The remaining strategies could be discontinuous, provided that the dynamics admit a well-defined solution.

\section{Application to non-renewable resource games}

In this section, we apply the above result to the problem of optimal management of a natural resource. This is a classical problem in the economics literature and has received much attention during the last 20 years; see Levhari and Mirman (1980), Clemhout and Wan (1985), Benhabib and Radner (1992) and Sorger (1998). The problem is generally cast in the infinite-horizon framework. When the problem is autonomous, this framework leads to consider stationary strategies which facilitate the analysis.

Now suppose that $N$ agents exploit on a bounded time horizon a non-renewable resource which is of common property. The evolution of the resource obeys the differential equation

$$
\dot{x}=-\sum_{i=1}^{N} u^{i}, \quad x(0)=x_{0}>0,
$$


where $x_{0}$ is the total quantity of the resource. The rate of depletion for player $i$ is $u^{i}$ which is assumed to be nonnegative. The payoff function is given by

$$
J_{i}\left(0, x_{0}, u\right)=\int_{0}^{T} \exp \left(-r_{i} t\right) L_{i}\left(u^{i}\right) \mathrm{d} t+\exp \left(-r_{i} T\right) S_{i}(x(T)), \quad 0<T<\infty,
$$

where $u=\left(u^{1}, \ldots, u^{N}\right)$. We consider feedback strategies $\phi^{i}$, admissible in the sense of Section 2. We need to add the condition $\phi(0, t)=0$ in view of the non-negativity constraints placed over the state variable and the controls of the players. The instantaneous utility function of the $i$ th player is $L^{i} ; r_{i} \geqslant 0$ and $S^{i}$ denote, respectively, the preference rate and the residual value of the resource.

We wish to show the existence of an MPNE of the game (13), (14) and study its qualitative properties. We suppose that all players have identical tastes and we look for a symmetric MPNE, $\hat{\phi}^{1}=\cdots=\hat{\phi}^{N}=\hat{\phi}$. Hence $L^{i}=L, S^{i}=S, r_{i}=r, \mathscr{E}_{i}=\mathscr{E}, \varphi_{i}=\varphi$ and $\mathscr{U}^{i}=\mathscr{U}, i=1, \ldots, N$. We will impose the following two hypotheses.

H1: $L$ is $\mathscr{C}^{3}$ on $(0, \infty)$, monotone increasing and strictly concave; $S$ is $\mathscr{C}^{2}$ on $(0, \infty)$, monotone increasing and concave.

By $\mathscr{E}$, we denote the inverse of the Arrow-Pratt index of risk aversion of the players: $\mathscr{E}(u)=-L^{\prime}(u) / L^{\prime \prime}(u)$. H1 implies $\mathscr{E}(u) \geqslant 0$.

H2: $\mathscr{E}(0)=0$ and $\mathscr{E} \prime(u) \leqslant N /(N-1)$.

For example, the homogeneous utility function $L(u)=u^{1+\gamma}, \gamma<0$, satisfies $\mathrm{H} 2$ whenever $-(N-1) / N<\gamma$. Let us observe that for this particular class of utility functions, when the last equality is binding, Eq. (15) reduces to $\hat{\phi}_{\tau}=r \mathscr{E}(\hat{\phi})$. Now we define the function $\varphi(x) \doteqdot\left(L^{\prime}\right)^{-1}\left(S^{\prime}(x)\right)$ which is obtained from $(5) ; \varphi$ gives the final value of the optimal control of the players. Taking into account the initial condition $\hat{\phi}(x, 0)=\varphi(x)$, the unique solution is $\hat{\phi}(x, \tau)=\exp (r \tau) \varphi(x)$. Note, however, that this solution is not admissible if $\varphi(0)>0$. In fact, if $r=0$ and $\varphi(0)>0$, there is no continuous and piecewise smooth solution to the equation. So we leave this case aside.

The assumption $\mathscr{E}(0)=0$ is essential for the fulfillment of the condition $\hat{\phi}(0, \tau)=0$. A utility function such as $L(u)=1-\exp (-\rho u), \rho>0$, does not meet the condition $\mathscr{E}(0)=0$.

Hypothesis $\mathrm{H} 2$ implies that the intertemporal rate of substitution of each player, $\mathscr{E}(u) / u$, is less than $N /(N-1)$. Hence the willingness to substitute consumption across time must be bounded by $N /(N-1)$. As we can see, the condition is more restrictive as the number of players increases and it is vacuous when $N=1$, i.e., in the optimal control problem. It can be shown that if this condition does not hold, then the unique solution (in a weak sense) of the Cauchy problem (15), (16) displayed below is discontinuous. This fact admits a simple interpretation: if the agents are not sufficiently risk averse or if the number of players is large, then a strong competition takes place in the extraction of the resource. The aim of adapting his or her strategy to the competitor's strategies in an optimum way motivates the discontinuity of the solution. The verification of optimality in this case is beyond the scope of this paper. 


\subsection{Analytical solution}

System (4) reduces to the equation

$$
\hat{\phi}_{\tau}+E(\hat{\phi}) \hat{\phi}_{x}=r \mathscr{E}(\hat{\phi}), \quad x>0, \tau>0 .
$$

The initial condition is

$$
\hat{\phi}(0, x)=\varphi(x), \quad x>0,
$$

where $E(u)=N u-(N-1) \mathscr{E}(u)$ and $\tau=T-t$. Let us observe that we have reversed time and that $\mathrm{H} 1$ implies that $\varphi$ is monotone increasing.

Problem (15), (16) was studied in Rincón-Zapatero et al. (1998), where the existence and uniqueness for a wide class of utility functions were established. However, the result does not apply if $\varphi(0)>0$, because in this case, $\hat{\phi}(0, T)=\varphi(0)>0$, so no solution of (15), (16) is admissible. For instance, the inequality $\varphi(0)>0$ happens when the residual function is linear, $S(x)=\alpha x$, because then $\varphi(x) \equiv k=\left(L^{\prime}\right)^{-1}(\alpha)$. Note that $\hat{\phi}(x, \tau) \equiv k$ is a solution to (15). This brings up the question of whether the discontinuous rule

$$
\phi(x, \tau)= \begin{cases}0 & \text { if } x=0, \\ k & \text { if } x>0\end{cases}
$$

is a Nash equilibrium (of course, in a more general class of policy functions than that considered here). As will be shown in Theorem 2, the hypotheses made on the model - together with the assumption $r=0$ whenever $\varphi(0)>0$ - imply the existence of a unique, continuous and piecewise smooth MPNE of the game.

In the proof of the following result, we will employ some concepts from the theory of quasi-linear partial differential equations of first order.

Theorem 2. Let us suppose that in the symmetric game (13), (14) hypotheses H1 and $\mathrm{H} 2$ hold. Then there exists a unique, symmetric MPNE $(\hat{\phi}, \ldots), \hat{\phi})$ in the two following cases:

(i) $\varphi(0)=0$ and $\lim _{u \rightarrow \infty} L^{\prime}(u)=0$.

(ii) $\varphi(0)>0$ and $r=0$.

Moreover, in case (ii) the optimal strategy satisfies the equation

$$
\hat{\phi}(x, t)=\varphi(x-(T-t) E(\hat{\phi}(x, t)))
$$

in the region $x \geqslant E(\varphi(0))(T-t)$, and it is given by

$$
\hat{\phi}(x, t)=E^{-1}\left(\frac{x}{T-t}\right)
$$

in the region $0 \leqslant x \leqslant E(\varphi(0))(T-t)$.

Proof. The first part was already proved in Rincón-Zapatero et al. (1998, Theorem 5.1). Thus we return to item (ii). Let us consider Eq. (15) associated with the symmetric 
game (13), (14) with $r=0$ :

$$
\begin{aligned}
& \hat{\phi}_{\tau}+E(\hat{\phi}) \hat{\phi}_{x}=0, \quad x>0, \quad \tau>0, \\
& \hat{\phi}(x, 0)=\varphi(x), \quad x>0,
\end{aligned}
$$

together with the admissibility condition

$$
\hat{\phi}(0, \tau)=0, \quad \tau \geqslant 0,
$$

where $\tau=T-t$. This is a mixed problem with boundary values which can be expressed as one of the initial values. To this end, we define $\hat{\phi}(x, 0)=0$, for all $x<0$. The characteristic system associated with (19) is given by

$$
\begin{gathered}
(\mathrm{d} / \mathrm{d} \tau) x=E(\hat{\phi}), \\
(\mathrm{d} / \mathrm{d} \tau) \hat{\phi}=0 .
\end{gathered}
$$

For $x>0$, the final condition (20) can be parameterized as

$$
\begin{aligned}
& \tau(\beta, 0)=0, \\
& x(\beta, 0)=\beta, \\
& \hat{\phi}(\beta, 0)=\varphi(\beta)
\end{aligned}
$$

with $\beta$ a positive parameter. It is clear that for all $\beta>0$ there exists a unique solution of (22), given by

$$
x(\beta, \tau)=\beta+E(\varphi(\beta)) \tau, \quad \hat{\phi}(\beta, \tau)=\varphi(\beta) .
$$

We can see that the solution $\hat{\phi}$ is constant along the characteristics $x(\beta, \tau)$. Hypothesis $\mathrm{H} 2$ implies that $E$ is strictly increasing in $[0, \infty)$, thus $E(\cdot)>0$, because $E(0)=0$. As a result, the straight lines $x(\beta, \tau)$ have positive slope. The solution along characteristics is well defined if and only if its envelope is empty; see Li Ta-Tsien (1994, Theorem 1.1). This is true if $(\partial / \partial \beta) x(\beta, \tau) \geqslant 0$. But

$$
\frac{\partial}{\partial \beta} x(\beta, \tau)=1+E^{\prime}(\varphi(\beta)) \varphi^{\prime}(\beta)>0
$$

because $E^{\prime}>0$ and $\varphi^{\prime}=\left(1 / L^{\prime \prime}\left(S^{\prime}\right)\right) S^{\prime \prime} \geqslant 0$. So we have a global, smooth solution of (19), in the region $\{(x, \tau): x \geqslant \tau E(\varphi(0)), \tau>0\}$ which is characterized by (17); see Li Ta-Tsien (1994). In Fig. 1, we sketch the characteristics in this region in the case where $S$ is a linear function. In the region $x \leqslant 0$ the unique solution of (22) is $x(\beta, \tau)=\hat{\phi}(\beta, \tau)=0$. Hence the solution in this region is identically null. There is a gap in the characteristics in the cone generated by the lines $x=0$ and $\tau=E(\varphi(0)) x$. It is well known that this cone can be filled with a rarefaction wave: $\hat{\phi}(x, \tau)=E^{-1}(x / \tau)$. H1 guarantees the existence of $E^{-1}$. The solution decreases continuously from $\varphi(0)$ to $E^{-1}(0)=0$ along the fan of straight lines shown in Fig. 1. It is clear that the solution 


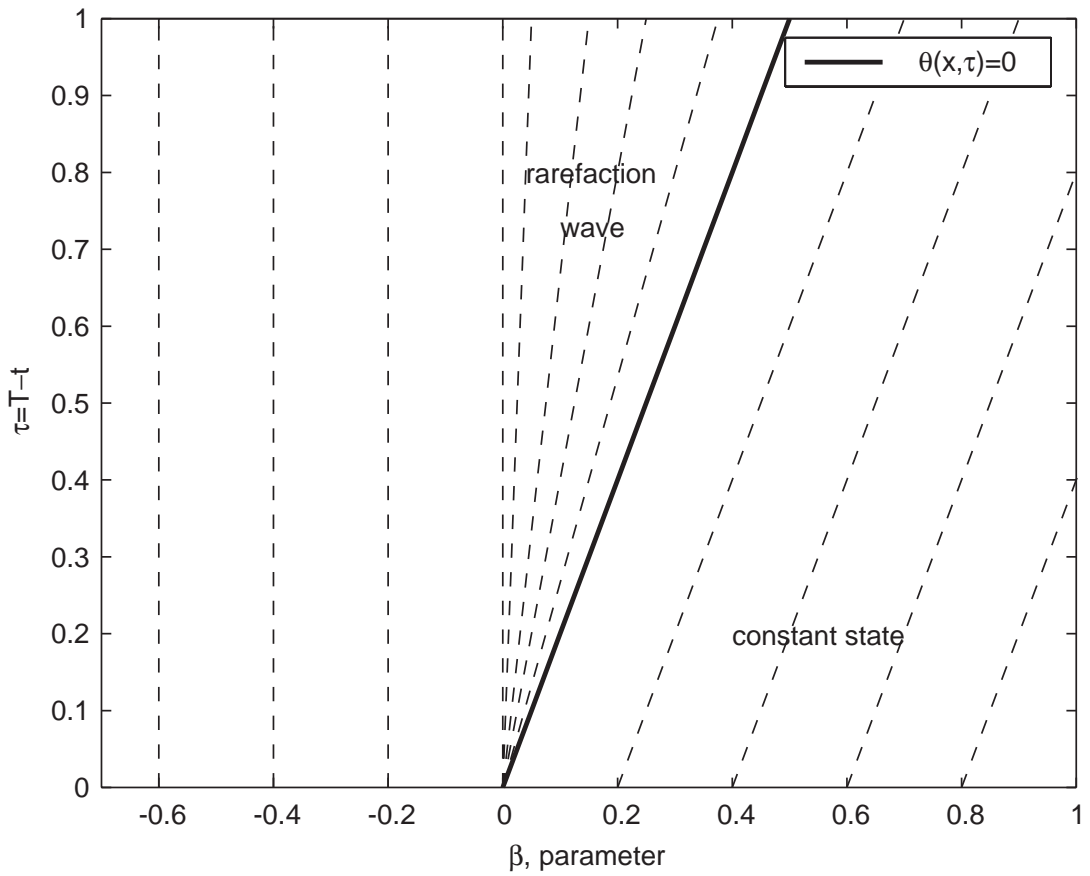

Fig. 1. Characteristics of the quasi-linear equation with linear residual function.

is continuous except at the unique point $(0,0)$ (when the game is finished), piecewise smooth and that $\hat{\phi}(0, \tau)=0$. In fact, $\hat{\phi}$ is locally Lipschitz with respect to $x$, so the trajectory associated with $\hat{\phi}$ is well defined (at least locally). Furthermore, $y^{\hat{\phi}}$ is convex with respect to $\tau$ - see Proposition 1 - hence it meets the curve of non-smoothness, $C=\{(x, \tau): \theta(x, \tau) \doteqdot x-\tau E(\varphi(0))=0\}$, in at most one point. We now show that this must happen in a non-tangential way.

Let $(x, \tau)$ belong to $C$. We then have

$$
\begin{aligned}
\theta_{x}(x, t) f(x, t, \hat{\phi}(x, t))+\theta_{t}(x, t) & =\theta_{x}(x, t)(-N \hat{\phi}(x, t))+\theta_{t}(x, t) \\
& =-N \varphi(0)+E(\varphi(0)) \\
& =-(N-1) \mathscr{E}(\varphi(0)) \neq 0 .
\end{aligned}
$$

In the second equality we have used the property that $\hat{\phi}(x, t)=\varphi(0)$ along $C$ and the third is due to the definition of $\mathscr{E}$ and $E$.

Uniqueness is a consequence of the construction of the solution.

To complete the proof, let us observe that the Hamiltonians of the players are strictly concave with respect to $u^{i}$, so according to (i) of Remark 1, any profile has the Nash property. As a consequence, Theorem 1 applies and $(\hat{\phi}, N), \hat{\phi})$ is an MPNE. 


\section{Remark 3.}

1. Actually, in case (i) or in case (ii) in the region $\{(x, \tau): x \geqslant E(\varphi(0)) \tau, \tau>0\}$, the solution is as smooth as $\varphi$. This is easily seen from the construction of the solution.

2. Direct application of Theorem 1.1 in Li Ta-Tsien (1994) requires that the initial condition $\varphi$ must be uniformly bounded. However, a careful review of the proof reveals that this restriction is superfluous in our problem because $E(\varphi(\beta))>0$ for all $\beta>0$.

3. When the residual function is linear, $S(x)=\alpha x$, then $\varphi \equiv k$, with $k=\left(L^{\prime}\right)^{-1}(\alpha)$, so $\hat{\phi} \equiv k$ on the region $x>E(k) \tau$. In this case the Markovian rules of the players are explicitly given by

$$
\hat{\phi}(x, t)= \begin{cases}k & \text { if } \frac{x}{T-t}>E(k), \\ E^{-1}\left(\frac{x}{T-t}\right) & \text { if } \frac{x}{T-t} \leqslant E(k) .\end{cases}
$$

The positive quadrant is partitioned into two different regions, with the solution being smooth in each one. In the region of the rarefaction wave, the solution decays from $\varphi(0)$ to zero. The extraction rate is constant along the rays in the fan of the characteristics. In Fig. 2 we show three different trajectories, depending on the initial condition. In the region of the rarefaction wave the extraction rate is decreasing along the optimal path.

Next, we study some qualitative aspects of the MPNE and the associated trajectory. To this end, let us define the sets

$$
\begin{aligned}
& X=\{(x, t): 0 \leqslant t<T, 0<x \leqslant N E(\varphi(0))(T-t)\}, \\
& Y=\{(x, t): 0 \leqslant t<T, x \geqslant N E(\varphi(0))(T-t)\} .
\end{aligned}
$$

Proposition 1. Let the hypotheses of Theorem 2 hold. Then

(i) $\hat{\phi}$ is increasing with respect to $x$.

(ii) The trajectory associated to $\hat{\phi}, y^{\hat{\phi}}(s ; x, t)$, is convex with respect to $s$.

Proof. As $E$ is increasing, $\hat{\phi}$ is also increasing in the rarefaction wave region $X$. Let $\tau>0$ be fixed and take $x>x^{\prime}$, with $(x, \tau),\left(x^{\prime}, \tau\right) \in Y$. Let $\beta, \beta^{\prime}$ be such that $x=x(\beta, \tau)$, $x^{\prime}=x\left(\beta^{\prime}, \tau\right)$. Since the slopes of the characteristics increase with the parameter $\beta$, we must have $\beta>\beta^{\prime}$, and hence $\varphi(\beta)>\varphi\left(\beta^{\prime}\right)$ because $\varphi$ is strictly increasing. Since $\hat{\phi}$ is constant along characteristics,

$$
\hat{\phi}(x, \tau)=\hat{\phi}(x(\beta, \tau), \tau)=\varphi(\beta)<\varphi\left(\beta^{\prime}\right)=\hat{\phi}\left(x\left(\beta^{\prime}, \tau\right), \tau\right)=\hat{\phi}\left(x^{\prime}, \tau\right) .
$$

Finally, if $\left(x^{\prime}, \tau\right) \in X$ and $(x, \tau) \in Y$, then the above reasoning also applies. This proves (i). 


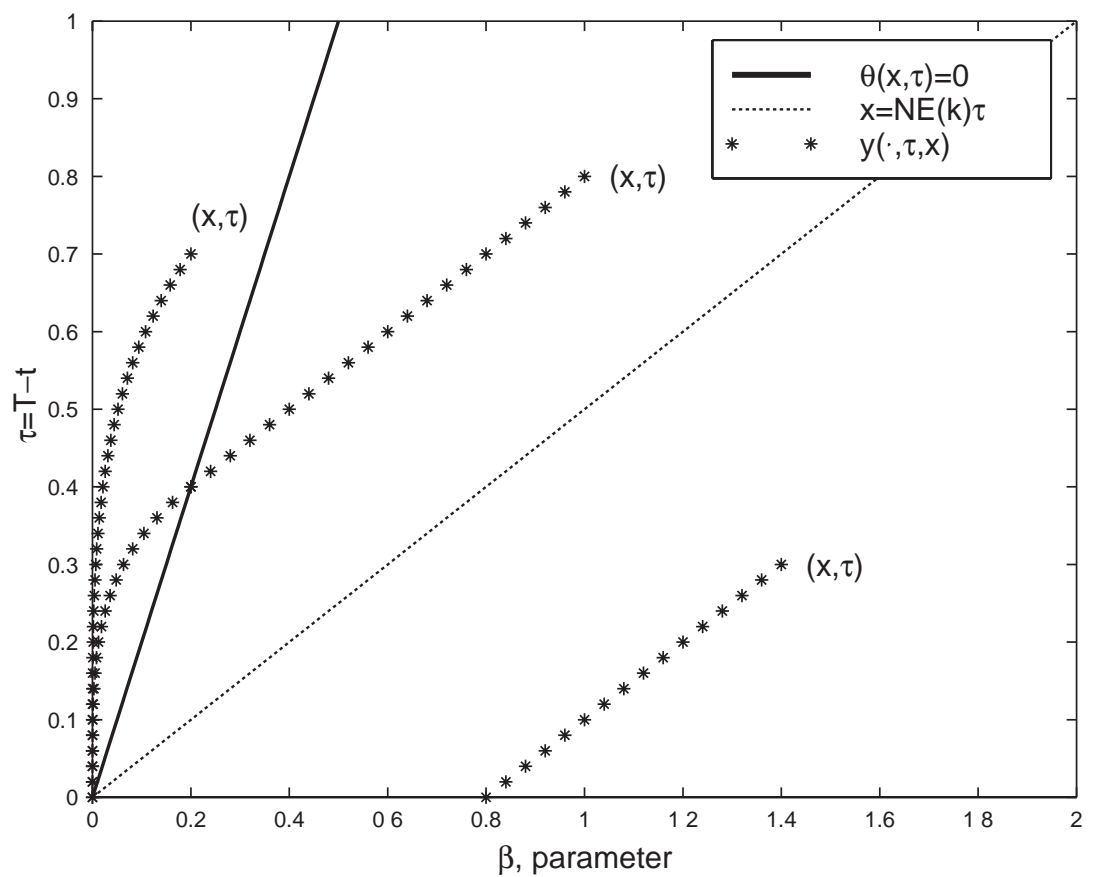

Fig. 2. Trajectories associated with MPNE for three different initial conditions.

With regard to (ii), when $y^{\hat{\phi}}$ is twice differentiable, we have

$$
\begin{aligned}
\frac{\mathrm{d}^{2} y^{\hat{\phi}}}{\mathrm{d} s^{2}}(s) & =-\frac{\mathrm{d}}{\mathrm{d} s}\left(N \hat{\phi}\left(y^{\hat{\phi}}(s), s\right)\right) \\
& =-N\left(\hat{\phi}_{x}\left(y^{\hat{\phi}}(s), s\right) \frac{\mathrm{d} y^{\hat{\phi}}}{\mathrm{d} s}(s)+\hat{\phi}_{t}\left(y^{\hat{\phi}}(s), s\right)\right) \\
& \left.=-N\left(-N \hat{\phi}\left(y^{\hat{\phi}}(s), s\right)\right) \hat{\phi}_{x}\left(y^{\hat{\phi}}(s), s\right)+\hat{\phi}_{t}\left(y^{\hat{\phi}}(s), s\right)\right) \\
& =N(N-1) \mathscr{E}\left(\hat{\phi}\left(y^{\hat{\phi}}(s), s\right)\right) \hat{\phi}_{x}\left(y^{\hat{\phi}}(s), s\right) \geqslant 0 .
\end{aligned}
$$

The fourth equality comes from (19), having taken into account $\tau=T-t$, the definition of the function $E(u)=N u-(N-1) \mathscr{E}(u)$ and item (i). We see that the facts that $y^{\hat{\phi}}$ is non-tangential to the set $C$ and that it is strictly decreasing assure that the points of intersection of the trajectory with $C$ are at most one. Henceforth the trajectory is twice differentiable except at most at a point, with non-negative second derivative. It turns out that it is convex on both time intervals. However, because $y^{\hat{\phi}}$ is differentiable everywhere with respect to $s$, then $y^{\hat{\phi}}$ is convex on $[t, T]$. 
It is interesting to analyze how much of the resource remains when the game ends. There exist initial conditions for which the resource is exhausted exactly at time $T$, whereas for other ones there remains a positive quantity of the resource at the end of the game. For the case when the salvage value of the players is linear, we can obtain more explicit results. Let us denote

$$
\begin{aligned}
& Y_{1}=\{(x, t): 0 \leqslant t<T, N E(k)(T-t) \leqslant x<N k(T-t)\}, \\
& Y_{2}=\{(x, t): 0 \leqslant t<T, x>N k(T-t)\} .
\end{aligned}
$$

Recall that when $S$ is linear, $\varphi \equiv k=\left(L^{\prime}\right)^{-1}(\alpha)$, and note that $Y_{1} \cup Y_{2}=Y$, where $Y$ was defined just prior to Proposition 1.

Proposition 2. Suppose that the assumptions of Theorem 2 hold and that $S(x)=\alpha x$. Then, for all $(x, t) \in X \cup Y_{1}, y^{\hat{\phi}}(T ; x, t)=0$ and for all $(x, t) \in Y_{2}, y^{\hat{\phi}}(T ; x, t)>0$.

Proof. Recall the characteristic system associated with (19)-(21):

$$
\begin{aligned}
& \frac{\mathrm{d}}{\mathrm{d} \tau} x=E(\hat{\phi}), \\
& \frac{\mathrm{d}}{\mathrm{d} \tau} \hat{\phi}=0
\end{aligned}
$$

with initial conditions

$$
x(\beta, 0)=\left\{\begin{array}{ll}
k & \text { if } \beta>0, \\
0 & \text { if } \beta<0,
\end{array} \quad \hat{\phi}(\beta, 0)=0, \quad \forall \beta \in \mathbb{R} .\right.
$$

The following reasonings all pertain to the $(x, \tau)$ plane. In $Y$, the optimal trajectories are parallel to the line $x=N k \tau$ which separates regions $X \cup Y_{1}$ and $Y_{2}$. Hence if the initial condition belongs to $X \cup Y_{1}$, then the optimal trajectory meets $X$ and converges to zero. On the other hand, if the initial condition belongs to $Y_{2}$, then the optimal trajectory is a straight line that intersects the $x$ axis at time $t=T$.

Next we study the dependence of the equilibrium with respect to the data of the model. The case $\varphi(0)=0$ was explored in Rincón-Zapatero et al. (1998, Corollaries 5.2 and 5.3). There the following were established for fixed values of time and the stock variable $x$ : (i) The extraction rate increases with the number of players whenever the intertemporal rate of substitution is less than 1. (ii) A higher intertemporal rate of substitution speeds up the extraction rate. It could be shown, with the same techniques, that the consumption rate also increases with the discount rate. Of course, this is not surprising because a higher discount rate makes the agent more indifferent about the future, given that the instantaneous utility of long-run consumption is weighted by an exponential factor decreasing in time. It is possible to reach similar conclusions for the game with $\varphi(0)>0$ but $r=0$. 
Proposition 3. Suppose that the assumptions of Theorem 2 hold. Then the following are true:

(a) Let $N^{\prime}<N^{\prime \prime}$ be two natural numbers. Then, for every fixed time $0 \leqslant t \leqslant T$ and stock value $x \in\left[0, x_{0}\right]$, the extraction rate $\hat{\phi}(x, \tau)$ is higher (lesser) in the game with $N=N^{\prime}$ than in the game with $N=N^{\prime \prime}$, whenever $\mathscr{E}(u) / u<1(\mathscr{E}(u) / u>1)$.

(b) Let $\mathscr{E}^{(1)}, \mathscr{E}^{(2)}$ be two positive, strictly increasing real functions of class $\mathscr{C}^{1}$, satisfying $\mathscr{E}^{(1)}(u) \geqslant \mathscr{E}^{(2)}(u)$ for all $u \geqslant 0$. Then, for every fixed time $0 \leqslant t \leqslant T$ and stock value $x \in\left[0, x_{0}\right]$, the extraction rate $\hat{\phi}(x, \tau)$ is higher in the game with $\mathscr{E}=\mathscr{E}^{(1)}$ than in the game with $\mathscr{E}=\mathscr{E}^{(2)}$.

(c) Let $S_{(1)}, S_{(2)}$ be two positive, strictly increasing real functions of class $\mathscr{C}^{2}$, satisfying $S_{(1)}(x) \leqslant S_{(2)}(x)$ for all $x>0$. Then, for every fixed time $0 \leqslant t \leqslant T$ and stock value $x \in\left[0, x_{0}\right]$, the extraction rate $\hat{\phi}(x, \tau)$ is higher in the game with $S=S_{(1)}$ than in the game with $S=S_{(2)}$.

Proof. We will prove the assertions of the proposition with the aid of Lemma A.1 in the Appendix. For part (a), take $f(u)=N^{\prime} u-\left(N^{\prime}-1\right) \mathscr{E}(u)$ and $g(w)=N^{\prime \prime} w-$ $\left(N^{\prime \prime}-1\right) \mathscr{E}(w)$. The condition $\mathscr{E}(u) / u<1(>1)$ implies $f \leqslant g(f \geqslant g)$ and we can apply Lemma A.1. For part (b), let $f(u)$ be the function $N u-(N-1) \mathscr{E}^{(1)}(u)$ and let $g(u)$ be the function $N u-(N-1) \mathscr{E}^{(2)}(u)$. It is clear that $f \leqslant g$; applying Lemma A.1 we reach the conclusion. Finally, for part (c), let us consider $\left.v_{0}(x)=\left(L^{\prime}\right)^{-1}\left(S_{(1)}^{\prime}\right)(x)\right)$ and $\left.w_{0}(x)=\left(L^{\prime}\right)^{-1}\left(S_{(2)}^{\prime}\right)(x)\right)$. It is evident that $w_{0} \leqslant v_{0}$ and that all the hypotheses of Lemma A.1 are fulfilled.

It is possible to prove that the MPNE of the game is inefficient. It is well known that, in general, the competition between two or more agents for the exploitation of the same resource stock leads to overconsumption. This is so because each player believes that the resource not consumed now could be used by the competitors. This common belief causes a rapid extraction of the resource.

Rincón-Zapatero et al. (2000) developed a general approach for derivation of efficient MPNE for two person differential games. The paper provides necessary and sufficient conditions. However, this approach will not be used here because we want to establish not only the inefficiency of the MPNE, but also the conditions under which the tragedy of commons applies. Let us assume that the agents determine their consumption rates in order to maximize the joint payoff. In the symmetric case, with $r=0$, the cooperative solution can be obtained from the solution of the optimal control problem

$$
\begin{gathered}
\max _{u \in \mathscr{U}} \int_{0}^{T} L(u) \mathrm{d} t+\alpha x(T) \\
\text { s.t: } \dot{x}=-N u, \\
x(0)=x_{0} .
\end{gathered}
$$


The partial differential equation and boundary conditions associated with the above problem are

$$
\begin{aligned}
& \phi_{\tau}^{\mathrm{p}}+N \phi_{x}^{\mathrm{p}} \phi^{\mathrm{p}}=r \mathscr{E}\left(\phi^{\mathrm{p}}\right), \quad x>0, \tau>0, \\
& \phi^{\mathrm{p}}(x, 0)=\varphi_{\mathrm{p}}(x), \quad x>0, \\
& \phi^{\mathrm{p}}(0, \tau)=0, \quad \tau>0,
\end{aligned}
$$

where $\varphi_{\mathrm{p}}(x)=\left(L^{\prime}\right)^{-1}\left(N S^{\prime}(x)\right)$. Here $\phi^{\mathrm{p}}$ stands for the Pareto optimal solution.

The following result can be proved in the same way as Theorem 2, hence it is stated without proof.

Theorem 3. Under hypothesis $\mathrm{H1}$, there exists a unique feedback solution of the control problem (29)-(31) in the class $\mathscr{U}$ in the two following cases:

(i) $\varphi^{\mathrm{p}}(0)=0$ and $\lim _{u \rightarrow \infty} L^{\prime}(u)=0$.

(ii) $\varphi_{\mathrm{p}}(0)>0$ and $r=0$.

Moreover, in case (ii) the optimal strategy satisfies the equation

$$
\phi^{\mathrm{p}}(x, t)=\varphi_{\mathrm{p}}\left(x-(T-t) N \phi^{\mathrm{p}}(x, t)\right)
$$

in the region $x \geqslant N \varphi_{\mathrm{p}}(0)(T-t)$ and it is given by

$$
\phi^{\mathrm{p}}(x, t)=\frac{x}{N(T-t)}
$$

in the region $x \leqslant N \varphi_{\mathrm{p}}(0)(T-t)$.

The line $x=N \varphi_{\mathrm{p}}(0)(T-t)$ separates the two regions of smoothness of $\phi^{\mathrm{p}}$. Neither the coefficient of risk aversion of the players nor the number of players have an effect on the existence of a Markov solution to the Pareto optimal control. This is due to the cooperation between the agents. Another observation is that, for any initial condition, the trajectory associated with $\phi^{\mathrm{p}}$ is a straight line; in fact, the cooperative trajectories coincide with the characteristics of the quasi-linear equation $\phi_{\tau}^{\mathrm{p}}(x, \tau)+N \phi^{\mathrm{p}}(x, t) \phi_{x}^{\mathrm{p}}(x, t)=0$. Furthermore, the optimal control is constant along the optimal trajectory. That is to say, the rate of extraction is constant under the cooperative management of the resource, in contrast with the non-cooperative case.

Proposition 4. Under the assumptions of Theorem 2, the MPNE of the game is inefficient and the tragedy of the commons apply, that is to say, $\phi^{\mathrm{p}}<\hat{\phi}$.

Proof. We only need to check that all conditions listed in Lemma A.1 are fulfilled in order to obtain the result. To this end we consider $f(v)=E(v)=N v-(N-1) \mathscr{E}(v)<N v=$ $g(v)$ and $v_{0}=\varphi>\varphi_{\mathrm{p}}=w_{0}$.

As a result, the competition between agents leads to depletion of the resource faster than in the cooperative case, giving rise to the tragedy of the commons. 


\subsection{Numerical solution}

The existence of a positive discount rate makes it difficult to find a closed-form solution to the game. Actually, it is not clear at the outset whether there is a global admissible solution to the quasi-linear equation. Therefore, it would be of interest to obtain - at least locally - numerical solutions in order to understand the behavior of the symmetric Nash equilibrium. There is considerable literature on numerical methods applied to quasi-linear equations. The monographs by Godlewski and Raviart (1991) or LeVeque (1992) provide a good account of appropriate methods. Given that we are looking for continuous solutions, we do not need to use the so-called conservative methods. This type of method is designed to approximate discontinuous solutions. We will use instead the simpler Courant-Isaacson-Rees (CIR) method. The idea is to make use of the information provided by the characteristic equations. We will describe briefly how this algorithm constructs a numerical solution. Given a Cauchy problem of one space dimension

$$
\begin{aligned}
& u_{\tau}(x, \tau)+f(u(x, \tau)) u_{x}(x, \tau)=g(u(x, \tau)), \quad x \in \mathbb{R}, \quad \tau \geqslant 0, \\
& u(x, 0)=\varphi(x),
\end{aligned}
$$

we discretize the $(x, \tau)$ plane by choosing a mesh width $h$ and a time step $k$. The approximate solution is to be computed at the mesh points $(i h, j k)$, for $i=\ldots,-1,0,1$, $2, \ldots, j=0,1,2, \ldots$ We let $U_{i}^{j}$ approximate the values of the true solution $u(i h, j k)$. As initial data for the numerical method we use $\varphi$ to define $U_{i}^{0}$. The characteristics of the equation in the $(x, \tau)$ plane are

$$
\begin{gathered}
\frac{\mathrm{d} x}{\mathrm{~d} \tau}=f(u), \\
\frac{\mathrm{d} u}{\mathrm{~d} \tau}=g(u) .
\end{gathered}
$$

The CIR method consists of solving certain equations along the characteristics going back from the point $(i h,(j+1) k)$. Given that the exact path of the characteristic is not known, it is approximated by a straight line with slope $f\left(U_{i}^{j}\right)$. This yields a value of $x$ at $\tau=j, i h-f\left(U_{i}^{j}\right) k$, which is not in general in the mesh grid. Hence the method uses linear interpolation between the two nearest mesh points, which are $\left(U_{i-1}^{j}, U_{i}^{j}\right)$ if the characteristic speed, $f\left(U_{i}^{j}\right)$, is positive, or $\left(U_{i}^{j}, U_{i+1}^{j}\right)$ if it is negative. For example, if $f\left(U_{i}^{j}\right)$ is positive, then the value of the approximate solution at point $i h-f\left(U_{i}^{j}\right) k$, denoted now as $\tilde{U}_{i}^{j}$, is

$$
\tilde{U}_{i}^{j}=U_{i}^{j}-\frac{h}{k} f\left(U_{i}^{j}\right)\left(U_{i}^{j}-U_{i-1}^{j}\right) .
$$

The determination of $U_{i}^{j+1}$ is accomplished by means of the Euler method:

$$
U_{i}^{j+1}=\tilde{U}_{i}^{j}+k g\left(\tilde{U}_{i}^{j}\right) .
$$




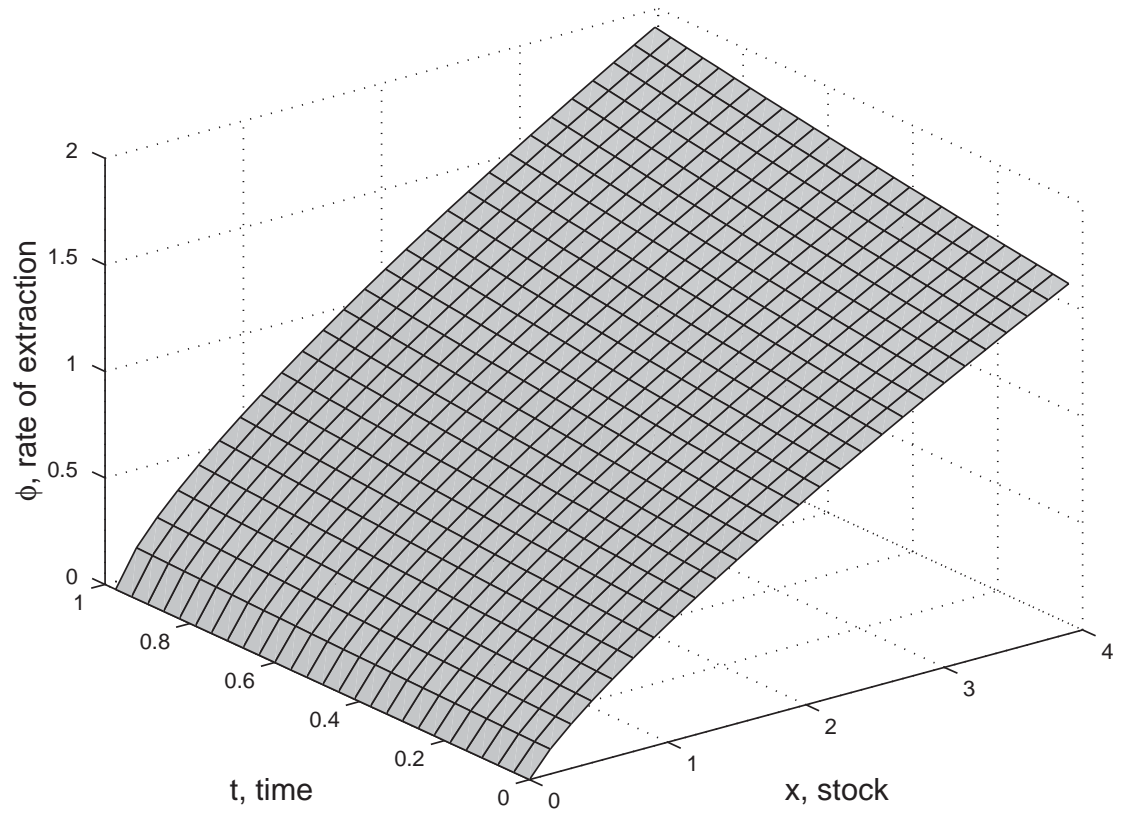

Fig. 3. The MPNE, $\hat{\phi}(x, t)$, in a smooth case.
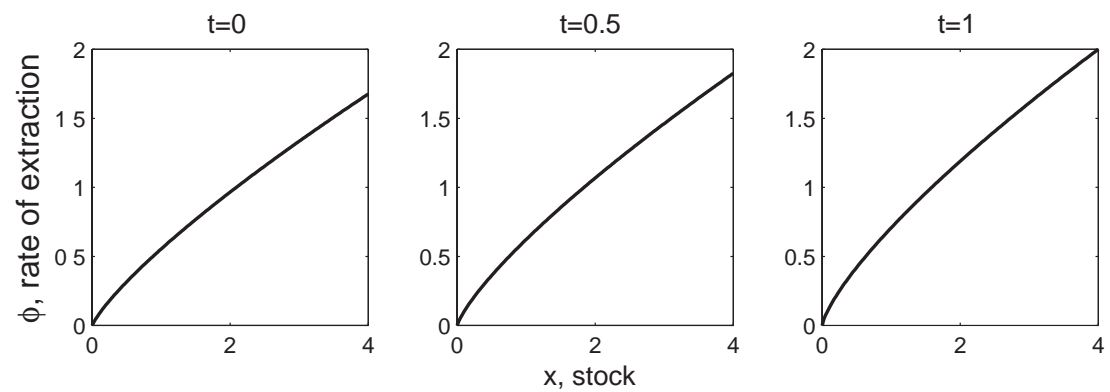

Fig. 4. The MPNE, $\hat{\phi}(x, t)$, in a smooth case at three different instants of time.

Of course, the algorithm also works - with obvious modifications - if $f$ and $g$ depend also both on time and the spatial variable. A necessary condition for the method to be useful is that $\left|(k / h) f\left(U_{i}^{j}\right)\right|<1$, which makes the linear interpolation possible.

We should say that the CIR method is not a good one for a problem involving discontinuous solutions. The trouble arises from the fact that there are multiple discontinuous solutions, that are defined in the weak sense, and the CIR method does not approximate, in general, the correct one. There are many experimental confirmations of this fact. 


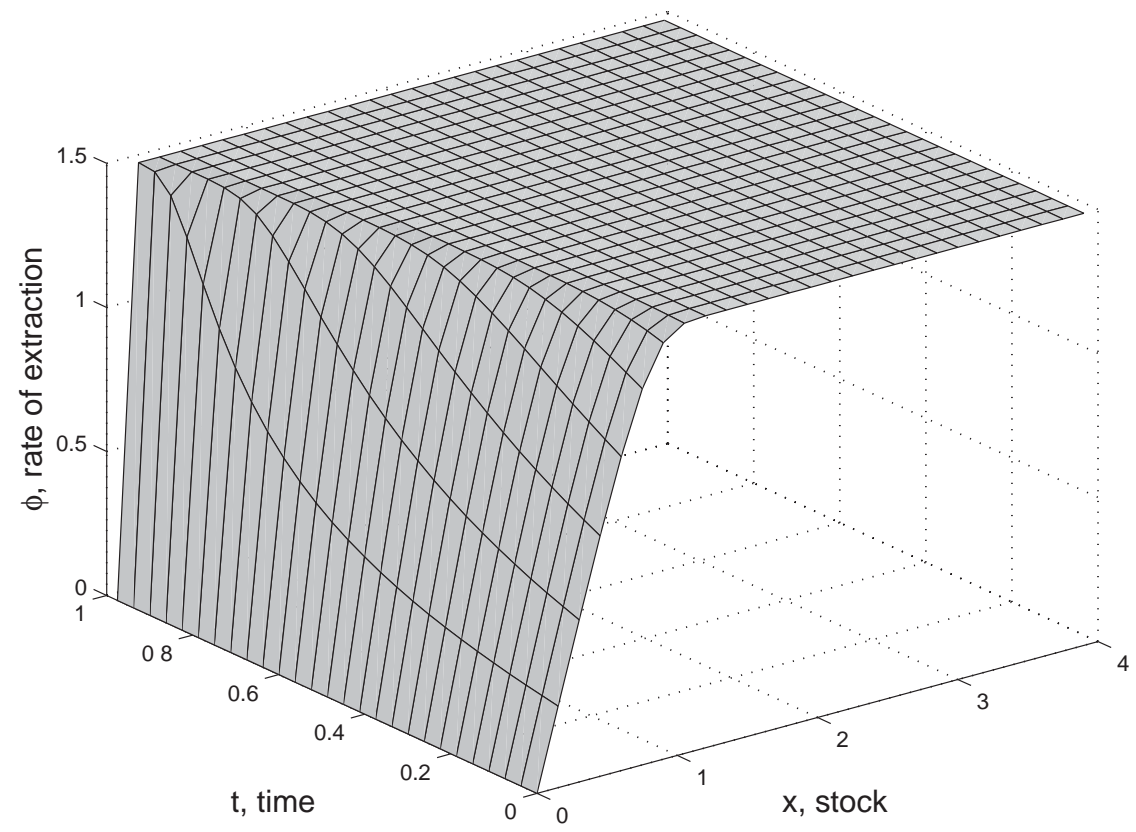

Fig. 5. The MPNE, $\hat{\phi}(x, t)$, when the residual function is linear.

We have not been able to demonstrate theoretically the existence of a continuous and piecewise smooth solution for all the problems studied in this section. However, we suspect this to be the case, and hence will use the CIR method in the following. Furthermore, it turns out that in problems where the analytical solution is known, the agreement of the numerical and the true solution is very satisfactory.

We wish to apply the CIR method to the initial value problem

$$
\begin{aligned}
& \hat{\phi}_{\tau}+E(\hat{\phi}) \hat{\phi}_{x}=r \mathscr{E}(\hat{\phi}), \\
& \hat{\phi}(x, 0)=\varphi(x) .
\end{aligned}
$$

The algorithm with this data becomes

$$
\begin{aligned}
& \tilde{U}_{i}^{j}=U_{i}^{j}-\frac{h}{k} E\left(U_{i}^{j}\right)\left(U_{i}^{j}-U_{i-1}^{j}\right), \\
& U_{i}^{j+1}=\tilde{U}_{i}^{j}+k r \mathscr{E}\left(\tilde{U}_{i}^{j}\right)
\end{aligned}
$$

with initial values $U_{i}^{0}=\varphi(i h)$.

For the numerical experiments, we take the instantaneous utility function to be $L(u)=$ $u^{1 / 3}$, the number of players two and $T=1$. First, we compute the solution when there is no discount rate, $r=0$, for the residual function $S(x)=\sqrt{2 x} / 48$. In this case Theorem 1 shows the existence of a unique solution for all $T$. The computed rate of extraction is smooth, as it can be seen in Fig. 3. The surface is increasing in time and stock as it is expected to be. 

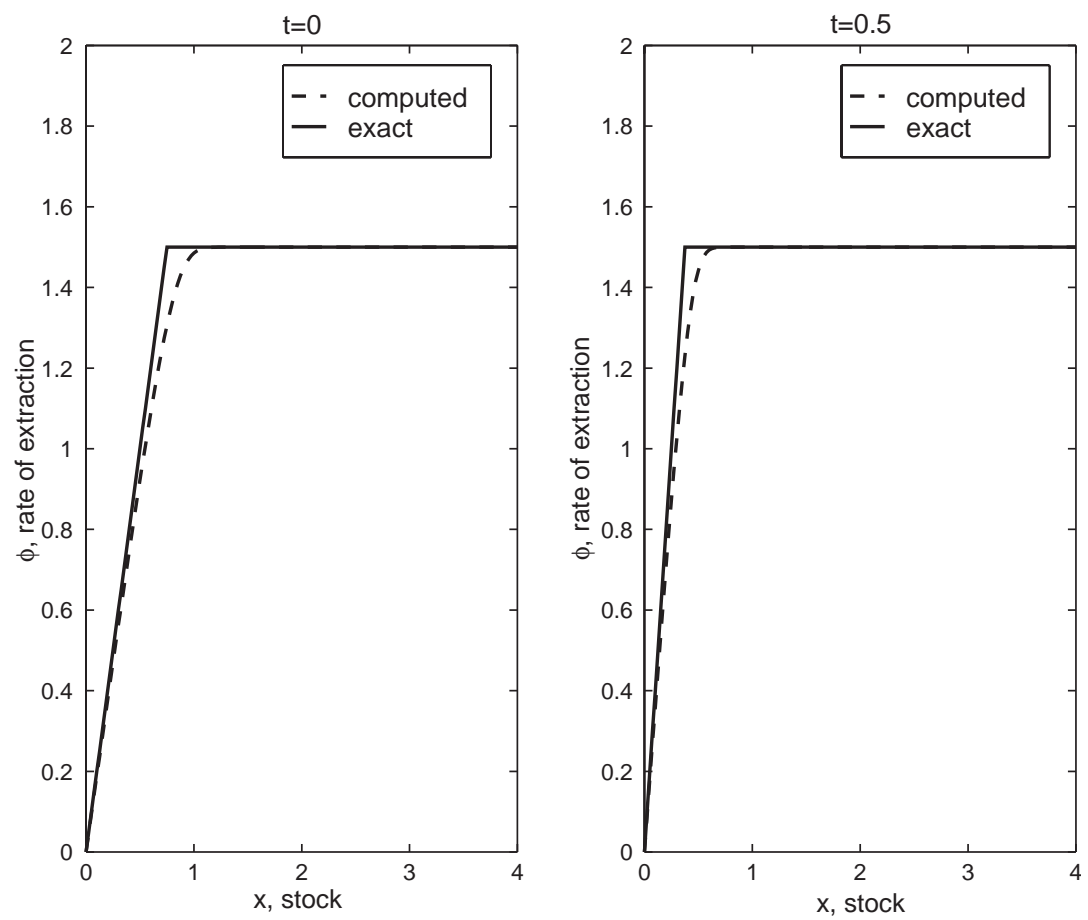

Fig. 6. Comparison of the exact and the computed solution at two different times.

Fig. 4 shows the profiles of the computed solution at three different instants of time.

Next we consider the case when the residual function is a linear function, $S(x)=a x$. Let us choose the constant $a$ such that $\hat{\phi}(x, 0)=3 / 2$ for $x>0$ - see the section above. The solution of (35), (36) is piecewise smooth. Fig. 5 depicts the computed rate of extraction and Fig. 6 provides a comparison of the numerical and the exact solutions at times $t=0$ and $t=0.5$. Note that the CIR method gives a smoother solution than the true one. This is typical of a first-order method.

The effect of discount on the rate of extraction is shown in Fig. 7. As the players become more impatient as their consumption increases.

\section{Concluding remarks}

This paper has introduced a new approach to the study of existence and uniqueness of MPNE, based on a quasi-linear system of partial differential equations. Usually, the HJB system associated with an $N$-person, non-zero sum differential game, exhibits non-linearities with respect to the gradients of the value functions. This fact makes it tedious to obtain results about the existence of solutions as well as to integrate the equations to find their analytical expressions. The same difficulties arise when we look for an approximate solution. 

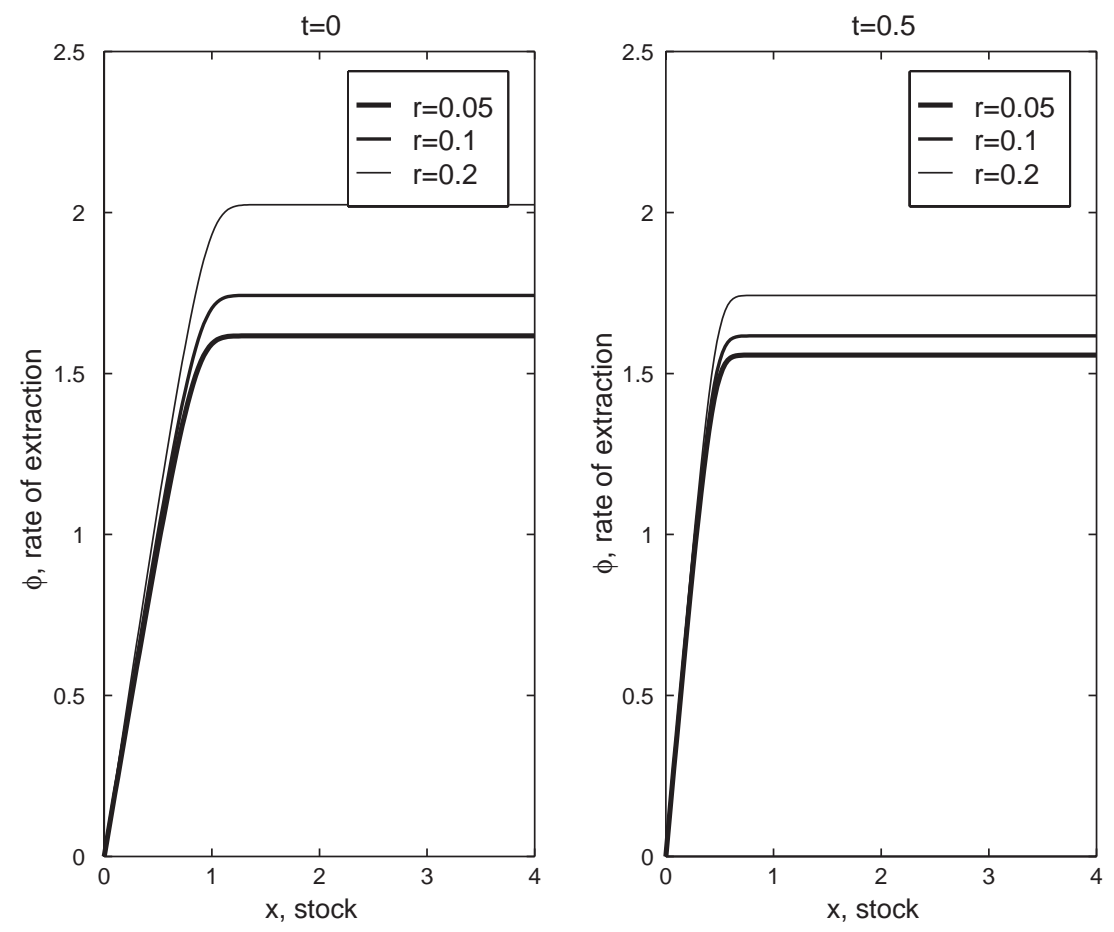

Fig. 7. Profiles of the MPNE at three instants of time for three different values of the discount factor.

The main idea brought forward in the paper is that in some cases it is possible to work with a system of quasi-linear PDEs where the unknown functions are the Markovian strategies of each player. The advantages of this system with respect to the HJB system is that now the gradient of the strategies enter in the equations in a linear way. This property makes the analysis easier.

The scope of our approach is limited to games where the dimension of the state space and the dimension of the control region of each player are the same. Furthermore, it is also necessary that the controls be interior to the control region. However, we hope that further research should allow us to extend this approach to a more general class of differential games. Constraints in the controls could be handled by incorporating penalty terms in the objective functional of the players.

Our approach is applied to non-renewable resource games with finite horizon and symmetric players, obtaining existence and uniqueness, whenever the discount rate is zero. Further research is needed to drop this assumption, to consider the asymmetric case, and to study the renewable resource case. It is likely that in some of the new situations listed above, discontinuous solutions appear; hence it will be of some interest to extend the theoretical framework to establish optimality of discontinuous solutions of the quasi-linear system. 


\section{Acknowledgements}

Financial support from Investigation Projects PB98-0393 of Dirección General de Enseñanza Superior e Investigación Científica and VA 108/01 of Junta de Castilla y León are gratefully acknowledged. The author is grateful to two anonymous referees for their comments and corrections. I am also indebted to the Associated Editor Tamer Başar for his remarks and for the careful reading of the manuscript. The author is responsible for any remaining error.

\section{Appendix A.}

Lemma 1. Consider the following Cauchy problems:

$$
(\mathrm{P} 1)\left\{\begin{array} { l } 
{ v _ { \tau } + f ( v ) v _ { x } = 0 , } \\
{ v ( 0 ) = v _ { 0 } , }
\end{array} \quad ( \mathrm { P } 2 ) \left\{\begin{array}{l}
w_{\tau}+g(w) w_{x}=0, \\
w(0)=w_{0} .
\end{array}\right.\right.
$$

Here $\tau \geqslant 0, x \in \mathbb{R}, v_{0}=w_{0}=0$ if $x<0$; the functions $f, g, v_{0}, w_{0}$ are $\mathscr{C}^{1}$, strictly monotone increasing, satisfying $f(0)=g(0)=0, w_{0}<v_{0}, f<g$, and with $v_{0}$, $w_{0}$ bounded. If $v, w$ are the solutions of ( $\mathrm{P} 1)$ and $(\mathrm{P} 2)$, respectively, then $w<v$.

Proof. The solutions to (P1) and (P2) are continuous and piecewise smooth, with two regions of smoothness separated by the lines $x=f\left(v_{0}(0)\right) \tau$ and $x=g\left(w_{0}(0)\right) \tau$, respectively. Let us consider two cases: (i) $f\left(v_{0}(0)\right)<g\left(w_{0}(0)\right)$ and (ii) $f\left(v_{0}(0)\right) \geqslant g\left(w_{0}(0)\right)$. In both cases, in the region where $v$ and $w$ are both given by rarefaction waves, $v(\tau, x)=f^{-1}(x / \tau)$ and $w(\tau, x)=g^{-1}(x / \tau)$. Hence, $v<w$ because $f<g$ and $f, g$ monotone increasing imply $g^{-1}<f^{-1}$. In case (i) let us denote

$$
\begin{aligned}
& \Omega_{1}=\left\{(x, \tau): 0 \leqslant \tau \leqslant T, f\left(v_{0}(0)\right) \tau \leqslant x \leqslant g\left(w_{0}(0)\right) \tau\right\}, \\
& \Omega_{2}=\left\{(x, t): 0 \leqslant \tau \leqslant T, g\left(w_{0}(0)\right) \tau \leqslant x\right\} .
\end{aligned}
$$

Let $(x, \tau) \in \Omega_{2}$ and, by way of contradiction, suppose that $v(x, \tau) \leqslant w(x, \tau)$. Let $\beta>0$, $\beta^{\prime}>0$ be such that the characteristics $x^{v}, x^{w}$ of (P1) and (P2) emanating from $(\beta, 0)$, $\left(\beta^{\prime}, 0\right)$, respectively, meet at point $(x, \tau)$. Then $v_{0}(\beta)=v(x, \tau) \leqslant w(x, \tau)=w_{0}\left(\beta^{\prime}\right)$, hence $f\left(v_{0}(\beta)\right) \leqslant f\left(w_{0}\left(\beta^{\prime}\right)\right)<g\left(w_{0}\left(\beta^{\prime}\right)\right)$. On the other hand,

$$
\beta+f\left(v_{0}(\beta)\right) \tau=x^{v}(\beta, \tau)=x=x^{w}\left(\beta^{\prime}, \tau\right)=\beta^{\prime}+g\left(w_{0}\left(\beta^{\prime}\right)\right) \tau .
$$

We obtain $\beta^{\prime}-\beta=\left(f\left(v_{0}(\beta)\right)-g\left(w_{0}\left(\beta^{\prime}\right)\right)\right) \tau<0$; therefore, $\beta^{\prime}<\beta$ and $w_{0}\left(\beta^{\prime}\right)<$ $w_{0}(\beta)<u_{0}(\beta)$, a contradiction. If $(x, \tau) \in \Omega_{1}$, then $w(x, \tau)=g^{-1}(x / \tau)<w_{0}(0)$. But $w_{0}(0)<v_{0}(0) \leqslant v(x, \tau)$, for all $(x, \tau) \in \Omega_{2}$, so we have the desired inequality.

In case (ii), let

$$
\begin{aligned}
& \Omega_{1}^{\prime}=\left\{(x, \tau): 0 \leqslant \tau \leqslant T, g\left(w_{0}(0)\right) \tau \leqslant x \leqslant f\left(v_{0}(0)\right) \tau\right\}, \\
& \Omega_{2}^{\prime}=\left\{(x, \tau): 0 \leqslant \tau \leqslant T, f\left(v_{0}(0)\right) \tau \leqslant x\right\} .
\end{aligned}
$$


For $(x, \tau) \in \Omega_{2}^{\prime}$, the reasoning proving the inequality is the same as in case (i) for $(x, \tau) \in \Omega_{2}$. When $(x, \tau) \in \Omega_{1}^{\prime}$ we have $v(x, \tau)=f^{-1}(x / \tau)$ and $w(x, \tau)=w_{0}(\beta)$ for suitable $\beta>0$. From the theory of conservation laws, we know that in the rarefaction wave region the value of $v$ is the same at points of the line connecting $(0,0)$ and $(x, \tau)$. Hence $v(x, \tau)=f^{-1}(x / \tau)=f^{-1}(m)$, where $1 / m$ is the slope of the above line (in the plane $(x, \tau)$ ) which verifies $1 / g\left(w_{0}(0)\right)<1 / m<1 / f\left(v_{0}(0)\right)$. The slope of the characteristic of equation $(\mathrm{P} 1)$ passing through points $(\beta, 0)$ and $(x, \tau)$ is $1 / g\left(w_{0}(\beta)\right)<1 / g\left(w_{0}(0)\right)<1 / m$. We then have

$$
w(x, \tau)=w_{0}(\beta)<g^{-1}(m)<f^{-1}(m)=v(x, \tau) .
$$

The first inequality follows because $g$ is increasing and the second one is a consequence of the fact that $f<g$ and $f, g$ are monotone increasing.

\section{References}

Başar, T., Olsder, G.J., 1999. Dynamic Noncooperative Game Theory. Society for Industrial and Applied Mathematics, Philadelphia, PA.

Benhabib, J., Radner, R., 1992. The joint exploitation of a productive asset: a game-theoretic approach. Economic Theory 2, 155-190.

Clemhout, S., Wan, Y.H., 1985. Dynamic common property resources and environmental problems. Journal of Optimization Theory and Applications 46, 471-481.

Fleming, W.H., Rishel, R.W., 1975. Deterministic and Stochastic Optimal Control. Springer, New York.

Friedman, A., 1971. Differential Games. Wiley, New York.

Godlewski, E., Raviart, P.A., 1991. Hyperbolic Systems of Conservation Laws. Mathématiques et Applications, Ellipses, Paris.

LeVeque, R.J., 1992. Numerical Methods for Conservation Laws. Birkhäuser, Basel.

Levhari, D., Mirman, L.J., 1980. The great fish war: an example using the Cournot-Nash solution. Bell Journal of Economics 11, 322-334.

Li Ta-Tsien, 1994. Global Classical Solutions for Quasilinear Hyperbolic Systems. Wiley, New York.

Rincón-Zapatero, J.P., Martínez, J., Martín-Herrán, G., 1998. New method to characterize subgame perfect Nash equilibria in differential games. Journal of Optimization Theory and Applications 96, 377-395.

Rincón-Zapatero, J.P., Martín-Herrán, G., Martínez, J., 2000. Identification of efficient subgame perfect Nash equilibria in a class of differential games. Journal of Optimization Theory and Applications 104, $235-242$.

Sorger, G., 1998. Markov-perfect Nash equilibria in a class of resource games. Economic Theory 11, $79-100$. 